\title{
Two-dimensional "transXend" detector for third-generation energy-resolved computed tomography
}

\section{AUTHOR(S):}

Kanno, Ikuo; Yamashita, Yoshiki; Kanai, Eri; Ogawa, Tsuyoshi; Shinsho, Kiyomitsu

\section{CITATION:}

Kanno, Ikuo ... [et al]. Two-dimensional "transXend" detector for third-generation energy-resolved computed tomography. Journal of Nuclear Science and Technology 2015, 53(2): 258-262

\section{ISSUE DATE:}

2015-04-23

URL:

http://hdl.handle.net/2433/203654

\section{RIGHT:}

This is an Accepted Manuscript of an article published by Taylor \& Francis in 'Journal of Nuclear Science and Technology' on 2016, available online: http://www.tandfonline.com/10.1080/00223131.2015.1037810.; The full-text file will be made open to the public on 29 April 2016 in accordance with publisher's 'Terms and Conditions for SelfArchiving'.; この論文は出版社版でありません。引用の際には出版社版をご確認ご利用ください。; This is not the published version. Please cite only the published version. 


\title{
ARTICLE
}

\section{Two-dimensional "transXend" detector for third-generation energy-resolved computed tomography}

\author{
Ikuo Kanno $^{\mathrm{a}^{*}}$, Yoshiki Yamashita ${ }^{\mathrm{a}, \dagger}$ Eri Kanai $^{\mathrm{a}}$, Tsuyoshi Ogawa ${ }^{\mathrm{a}, \sharp}$ and Kiyomitsu Shinsho ${ }^{\mathrm{b}}$ \\ ${ }^{\mathrm{a}}$ Graduate School of Engineering, Kyoto University, Nishikyo, Kyoto 615-8530, Japan; \\ ${ }^{b}$ Graduate School of Human Health Sciences, Tokyo Metropolitan University, Arakawa, Tokyo \\ 116-8551, Japan
}

The "transXend" detector measures X-rays as electric currents and provides the energy distribution of the measured X-rays after analysis. Capabilities of material distinction, effective atomic number measurement, and low-dose exposure CT with using high K-edge contrast agent from the use of the transXend detector in energy-resolved computed tomography (CT) have been demonstrated via the first-generation CT measurements. For application of the principle of the transXend detector to the third-generation CT for human subjects in future work, a method for fabrication of a two-dimensional transXend detector is proposed and demonstrated with using a commercial two-dimensional detector and two kinds of strip absorbers. The energy-resolved CT is performed with placing a proposed absorber system in front of a two-dimensional detector which is used for conventional current measurement CT.

Keywords: X-ray; energy distribution; unfolding; computed tomography; energy-resolved measurement

\footnotetext{
${ }^{*}$ Corresponding author. Email: kanno@nucleng.kyoto-u.ac.jp

${ }^{\dagger}$ Present address: Institute of Materials Structure Science, High Energy Accelerator Research Organization, Tsukuba, Ibaraki 305-0801, Japan

\$ Present address: Hitachi Works, Power Systems Company, Hitachi, Ltd., Hitachi, Ibaraki 317-8511, Japan.
} 


\section{Introduction}

The X-ray computed tomography (CT) is a powerful modality for finding tumors such as cancers. The X-ray CT measures the attenuation of X-rays by materials inside a subject and gives linear attenuation coefficient distribution in tomographic images. Because the linear attenuation coefficients of normal and cancer tissues are similar, iodine contrast agent is injected into a blood vessel: cancer tissue has higher iodine concentration than normal tissue, because the portion of blood vessel of cancer tissue is higher than that of normal tissue.

The mass attenuation coefficients of acrylic (a substitute for normal tissue) and iodine are shown in Figure 1 [1]. The calculated energy spectra of X-rays with using Birch's formula for the tube voltage 80 and $120 \mathrm{kV}$ are also shown in the figure. [2] In conventional X-ray CT, X-rays are measured as electric current. The measured electric current $I$ is written as follows,

$$
I \propto \int E \cdot Y(E) d E
$$

Here, $E$ is the energy of X-rays and $Y(E)$ is the number of X-rays with their energy $E$. Because the absorption K-edge of iodine is in $33.2 \mathrm{keV}$ in energy, the $\mathrm{X}$-ray attenuation effect by iodine is more legible with the tube voltage $80 \mathrm{kV}$ than $120 \mathrm{kV}$. In the same way, the effect of iodine attenuation is less obvious for thick subject than thin one, because higher energy X-rays dominate more after passing a thicker subject. This phenomenon is called a beam hardening effect. [3]

Energy-resolved computed tomography (CT) uses X-rays within a specific energy range of interest. The attenuation effect of iodine can be estimated using X-rays in the energy range, for example, 33.5-40 keV. In energy-resolved CT, the attenuation of X-rays by certain materials is observed, regardless of the thickness of the subject and the X-ray tube voltage used. Energy-resolved CT is also immune to the beam hardening effect, while conventional current measurement CT suffers from this effect.

Energy measurement of each X-ray, i.e., photon counting, is an ideal method for 
energy-resolved CT. However, the number of X-rays arriving at the detector ranges from $10^{6}$ to $10^{9} \mathrm{~mm}^{-2} \mathrm{~s}^{-1}$ and is far too high for photon counting to be performed [4]. The development of a high-counting-rate X-ray detector has been a focus of research to enable practical application of energy-resolved CT.

The authors invented a new detector system, the "transXend" detector, for energy-resolved CT [5]. The transXend detector consists of several detector segments that are aligned in the direction of the X-rays. The transXend detector measures the X-rays as electric currents and provides the energy distributions of the X-rays after analysis, using previously obtained response functions.

Using the proposed transXend detector, we demonstrated the advantages of energy-resolved CT: enhanced iodine contrast [6], material distinction between iodine and calcium [7], effective atomic number measurements [8], and low-dose CT with a high K-edge contrast agent [9]. The measurements were, however, performed using a first-generation CT apparatus: pencil X-ray beams were measured using a detector with translation and rotation movements only. To apply the principle of the transXend detector to human patients, CT measurements should be performed using a third-generation CT apparatus, where fan- or cone-shaped X-ray beams are measured using two-dimensional detectors with rotational movement.

It may be possible to arrange conventional stacked transXend detectors, which is described in Kanno et al. [5], in two dimensions; however, the number of read-out circuits required for this would be enormous and dead space between the neighboring transXend detectors would be a problem. Along with these disadvantages, the spatial resolution would be insufficient, because the segment detectors cannot be made small enough.

In this paper, a method to apply the principle of the transXend detector to the third-generation CT with using a two-dimensional detector and two kinds of strip absorbers is described. With placing strip absorbers in front of a two-dimensional detector, such as a flat 
panel detector in a hospital, energy-resolved CT would be performed.

\section{Two-dimensional transXend Detector}

\subsection{Principle}

We propose a transXend detector system using a commercially available two-dimensional detector and two types of strip absorber. The two-dimensional detector can be a flat panel detector, a thermo-luminescent (TL) plate, or an image plate (IP). While the TL plate and the IP are integrating-type detectors, they can be used for CT measurement in case of observation of an axially-symmetric subject. In this paper, a TL plate was employed for demonstrating the operating principle of the two-dimensional transXend detector.

As shown in Figure 2, strip absorbers A and B are arranged from top to bottom and from right to left with a set spacing between them. A two-dimensional detector is then placed behind these absorbers. An enlarged portion of the figure shows four different regions, $(\alpha)$, $(\beta),(\gamma)$, and $(\delta)$, in the two-dimensional detector. In region $(\alpha)$, only X-rays that passed through the air arrive. Similarly, X-rays that passed through the absorbers A, B and A+B arrive at regions $(\beta),(\gamma)$ and $(\delta)$, respectively. By using 0.1-mm-thick tin and copper for absorbers A and B, respectively, the X-ray spectra arriving at each region are calculated as shown in Figure 3 for the case of $120 \mathrm{kV}$ tube voltage with tungsten target [2]. The pixels of the two-dimensional detector in each region have the roles of segment detectors. By unfolding the electric current, i.e., thermo-luminescence in case of TL plate, measured by the pixels in the four regions, X-ray energy distributions can be obtained for the $(x, y)$ positions in the two-dimensional detector.

\subsection{Experiments}

The TL plate dimensions are $80 \times 80 \times 1 \mathrm{~mm}^{3}$. The specifications of the TL plate are reported by Shinsho et al. [10]. The tin and copper absorbers are $0.5 \mathrm{~mm}$ wide, $0.1 \mathrm{~mm}$ thick 
and $50 \mathrm{~mm}$ long. The tin and copper absorbers are placed on separate 1-mm-thick acrylic plates, with spacing between the neighboring absorbers of $0.5 \mathrm{~mm}$. The two acrylic plates with the different absorbers are attached to each other with the geometry shown in Figure 2.

The experimental setup is similar to that shown in Figure 1 of Yamashita et al. [9]. The X-ray tube used is the ERESCO 42 MF4 (GE Sensing \& Inspection Technologies, Ahrensburg, Germany) with a tungsten target. The X-ray tube has built-in filters made from 0.8-mm-thick beryllium and 2-mm-thick aluminum. The tube voltages used are 100, 120 and $150 \mathrm{kV}$. The tube current and the exposure time are $6 \mathrm{~mA}$ and $60 \mathrm{~min}$, respectively.

Response function measurements were performed using a diluted iodine tincture with thicknesses ranging from 0 to $60 \mu \mathrm{m}$ at intervals of $15 \mu \mathrm{m}$. To avoid the influence of fading effect, the thermo-luminescence was measured $1 \mathrm{~h}$ after irradiation. The thermo-luminescence measurements were performed with the measurement system developed by Shinsho et al. [11]: the TL plate was placed on a computer-controlled heater and was heated up to $300^{\circ} \mathrm{C}$ at a rate of $1^{\circ} \mathrm{C} \cdot \mathrm{s}^{-1}$. The thermo-luminescence was measured using a charge-coupled device camera (ATIK 383L+, Norwich, UK) with pixel size of $5.4 \times 5.4 \mu \mathrm{m}^{2}$. The procedure described above was repeated while adding 10-mm-thick acrylic plates up to a limit of 30 $\mathrm{mm}$.

CT measurements were performed for a 30-mm-diameter cylindrical acrylic phantom. The phantom had a 5-mm-diameter hole at its center. This hole was filled with the diluted iodine tincture with effective iodine thickness of $30 \mu \mathrm{m}$ for a 5-mm X-ray path. The measured thermo-luminescence data were duplicated 17 times to provide CT data with 10 degrees of rotation.

\subsection{Analysis}

A thermo-luminescence image of the cylindrical phantom and the strip absorbers is shown in Figure 4. In the lattice structure, four different regions can be observed. The X-ray 
attenuation effect of the iodine is shown at the center of the figure. The number of photons averaged for the $2 \times 2$ pixels at the center of each region is used as the foreground data. These data were taken along a virtual horizontal line with avoiding noises shown as some white and black dots. The thermo-luminescence efficiency of the corresponding position is obtained as the number of photons observed after X-ray exposure without placement of the phantom. The net photon number for each region of the TL plate is estimated by dividing the foreground data by the thermo-luminescence efficiency.

Using the net photon numbers in the neighboring $(\alpha)-(\delta)$ regions, the X-ray energy distributions can be unfolded using the SAND-II code [12] with the assigned energy ranges shown in Table 1. Because the measured maximum X-ray energy showed that the actual tube voltage is less than the indicated voltage, the maximum energies in energy range $E_{4}$ for each tube voltage are assigned smaller than nominal values of 96, 114 and $143 \mathrm{keV}$ for the tube voltages of 100, 120 and $150 \mathrm{kV}$, respectively.

\section{Results and Discussion}

CT images are shown in Figure 5 for the tube voltage of $150 \mathrm{kV}$ obtained by (a) energy-resolved CT using the X-rays in energy range $E_{2}$, and (b) conventional current measurement CT measured at region $(\alpha)$. The image reconstructions are performed using the filtered back-projection algorithm [13]. The legible artifacts in radial direction are caused by the small number of rotation data. These artifacts will not be obvious with increasing the number of rotations. The CT value profiles are summarized in Figure 6. Under cone beam exposure, the shape of the phantom is enlarged. The current measurement CT profiles are shown as negative values to provide a clear comparison.

The CT values of the acrylic and iodine regions are averaged for ten and three measured points, i.e., the points from 9.5 to $19 \mathrm{~mm}$, and from -0.95 to $0.95 \mathrm{~mm}$ in Fig. 6, respectively. Obtained results are plotted in Figure 7 for acrylic (squares), iodine (circles) 
measured by energy-resolved (solid) and current (open) CT methods. In current measurement CT, the CT values of iodine decrease as the tube voltage increases. This is a result of the beam hardening effect. In contrast, the values obtained by energy-resolved CT remain fairly consistent within the error bars. These unchanging results are the evidence that energy-resolved CT has been performed successfully.

\section{Conclusion}

For future application of the transXend detector principle to human bodies, a two-dimensional transXend detector was demonstrated using a thermo-luminescent plate and two types of strip absorbers. The CT value differences obtained between iodine and acrylic regions showed constant values in energy-resolved CT, while they decreased with increasing tube voltage in conventional current measurement CT.

For on-line readout of the two-dimensional transXend detector, we intend to apply the method described in this paper with a Si-based flat panel detector.

\section{Acknowledgements}

The authors would like to thank Prof. K. Hitomi, Tohoku University, for allowing them to use his image reconstruction program. This work was supported by the Japan Society for the Promotion of Science with a Grant-in-Aid for Scientific Research. 


\section{References}

[1] Hubbell JH, Seltzer SM. Tables of X-ray mass attenuation coefficients and mass energy-absorption coefficients from $1 \mathrm{keV}$ to $20 \mathrm{MeV}$ for elements $\mathrm{Z}=1$ to 92 and 48 additional substances of dosimetric interest. The Physical Measurement Laboratory, The National Institute of Standard and Technology; 2010. Available from: http://www.nist.gov /pml/data /xraycoef / index.cfm.

[2] Birch R, Marshall M. Computation of Bremsstrahlung x-ray spectra and comparison with spectra measured with aGe(Li) detector. Phys Med Biol. 1979;24:505.517.

[3] Brooks RA, Di Chiro G. Beam hardening in X-ray reconstructive tomography. Phys Med Biol. 1976;21:390-398.

[4] Xu C, Danielsson M, Karlsson S, Svensson C, Bornefalk H. Preliminary evaluation of a silicon strip detector for photon-counting spectral CT. Nucl Instrum Methods Phys Res A. 2012;677:45-51.

[5] Kanno I, Imamura R, Mikami K,Uesaka A, Hashimoto M, Ohtaka M, Ara K, Nomiya S, Onabe H. A current mode detector for unfolding X-ray energy distribution. J Nucl Sci Technol. 2008;45:1165-1170.

[6] Imamura R, Mikami K, Minami Y, Kanno I, Ohtaka M, Hashimoto M, Ara K, Onabe H. Unfolding method with X-ray path length-dependent response functions for computed tomography using X-ray energy information. J Nucl Sci Technol. 2010;47:1075-1082.

[7] Kanno I, Imamura R, Yamashita Y, Ohtaka M, Hashimoto M, Ara K, Onabe H. Using energy-resolved X-ray computed tomography with a current mode detector to distinguish materials. Jpn J Appl Phys. 2014; 53: 056601-4.

[8] Yamashita Y, Kimura M, Kitahara M, Hamaguchi T, Kanno I, Ohtaka M, Hashimoto M, Ara K, Onabe H. Measurement of effective atomic numbers using energy-resolved computed tomography. J Nucl Sci Technol. 2014;51:1256-1263.

[9] Yamashita Y, Shima K, Kanno I, Ohtaka M, Hashimoto M, Ara K, Onabe H. Low-dose 
exposure energy-resolved X-ray computed tomography using a contrast agent with a high-energy K-edge. J Nucl Sci Technol. 2014; 51: 91-97.

[10] Shinsho K, Kawaji Y, Otsubo K, Koba Y, Wakabayashi G, Matsumoto K, Ushiba H. Thermoluminescence properties of commercial $\mathrm{Al}_{2} \mathrm{O}_{3}$ ceramics plate (Kyocera corp., A476). Rad Meas., submitted.

[11] Shinsho K, Koba Y, Wakabayashi G, Tamatsu S, Fukuda S, Morimoto R, Maruyama D, Saitoh H, Sakurai N. Basic characteristics of tissue-equivalent phantom thermoluminescence slab dosimeter using new TL phosphor Li3B7012:Cu. Rad Meas. 2014; 62: 15-21.

[12] McElroy W, Berg S, Crockes T, Hawkins G. A computer-automated iterative method for neutron flux spectra determination by foil activation. New Mexico: Air Force Weapons Laboratory; $1967 . \quad$ p.8-16.

[13] Shepp LA, Logan BF. Fourier reconstruction of a head section. IEEE Trans Nucl Sci 1974; NS21: 21-43. 


\section{Figure captions}

Table 1. Assigned energy ranges. $E_{\max }$ is described in the text.

Figure 1 Mass attenuation coefficients of iodine and acrylic as a function of photon energy, as well as the calculated X-ray energy spectra with the tube voltages 80 (thin line) and 120 (thick line) $\mathrm{kV}$.

Figure 2 Schematic of two-dimensional transXend detector made from a thermo-luminescent plate and two types of strip absorbers. X-rays with four different energy spectra enter the thermo-luminescent plate after passing through $(\alpha)$ the air, $(\beta)$ absorber $A$, $(\gamma)$ absorber B, and $(\delta)$ absorbers A and B.

Figure 3 Calculated X-ray energy spectra for tube voltage of $120 \mathrm{kV}$ after passing through the air (thick solid line), 0.1-mm-thick tin (thin solid line), 0.1-mm-thick copper (thick broken line) and both 0.1-mm-thick tin and copper (thin broken line).

Figure 4 Thermo-luminescence image. The black area in the center corresponds to the iodine region in the acrylic phantom.

Figure 5 CT images obtained by (a) energy-resolved CT with X-rays in the energy range $E_{2}$, and (b) current CT. The tube voltage was $150 \mathrm{kV}$. The CT values are shown in terms of the linear attenuation coefficient $\left(\mathrm{cm}^{-1}\right)$.

Figure 6 CT value profiles obtained by energy-resolved CT and current CT with tube voltages of 100 (solid line), 120 (broken line) and 150 (dotted line) kV. Current CT results are shown as negative values for clear comparison. The CT values are shown in terms of the linear attenuation coefficient $\left(\mathrm{cm}^{-1}\right)$.

Figure 7 Summary of the CT values of acrylic (squares), iodine (circles) measured by energy-resolved (solid) and current (open) CT methods. The CT values are shown in terms of the linear attenuation coefficient $\left(\mathrm{cm}^{-1}\right)$. 
Table 1

\begin{tabular}{ccccc}
\hline & $E_{1}$ & $E_{2}$ & $E_{3}$ & $E_{4}$ \\
\hline $\mathrm{keV}$ & $15.0-33.5$ & $33.5-39.0$ & $39.0-80.0$ & $80.0-E_{\max }$ \\
\hline
\end{tabular}




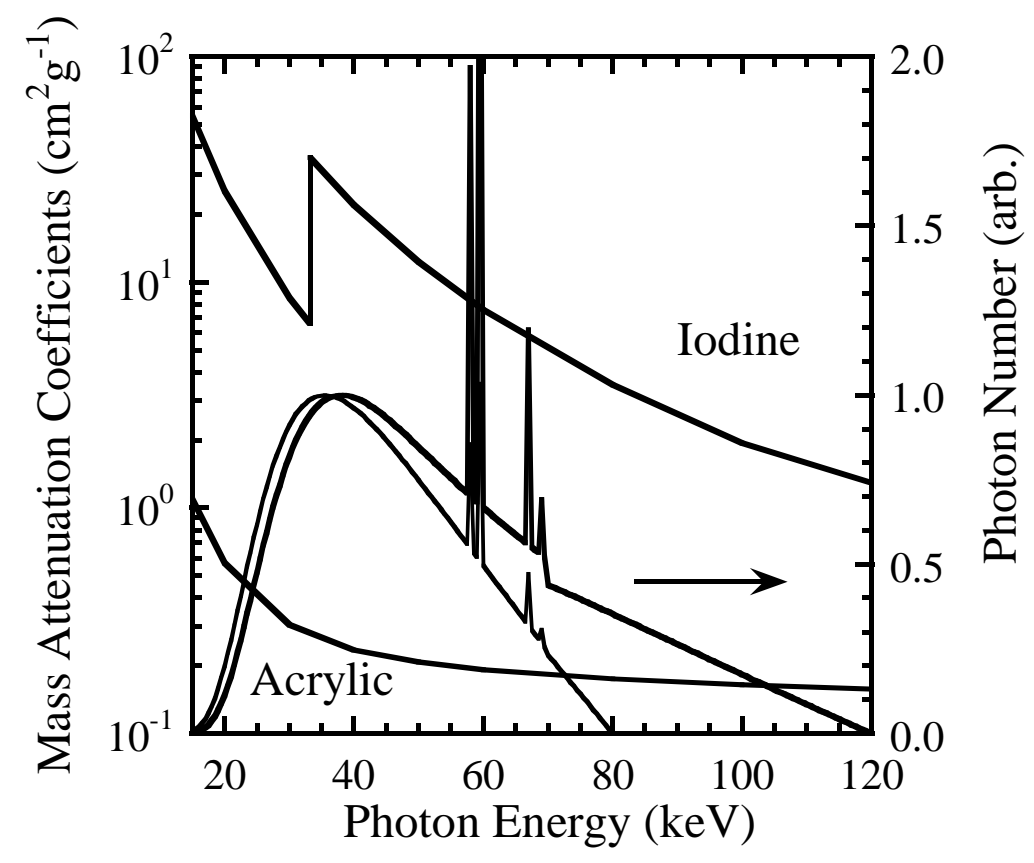

Figure 1 


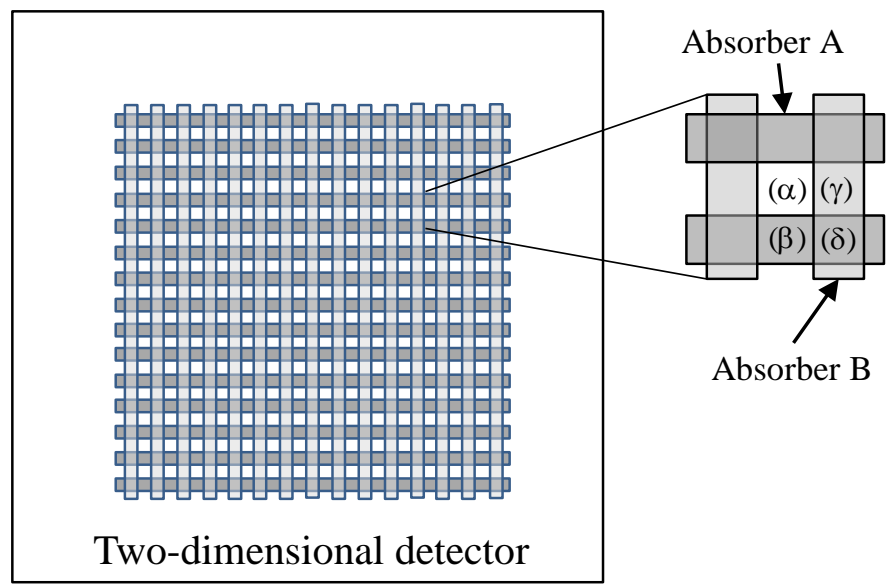

Figure 2 


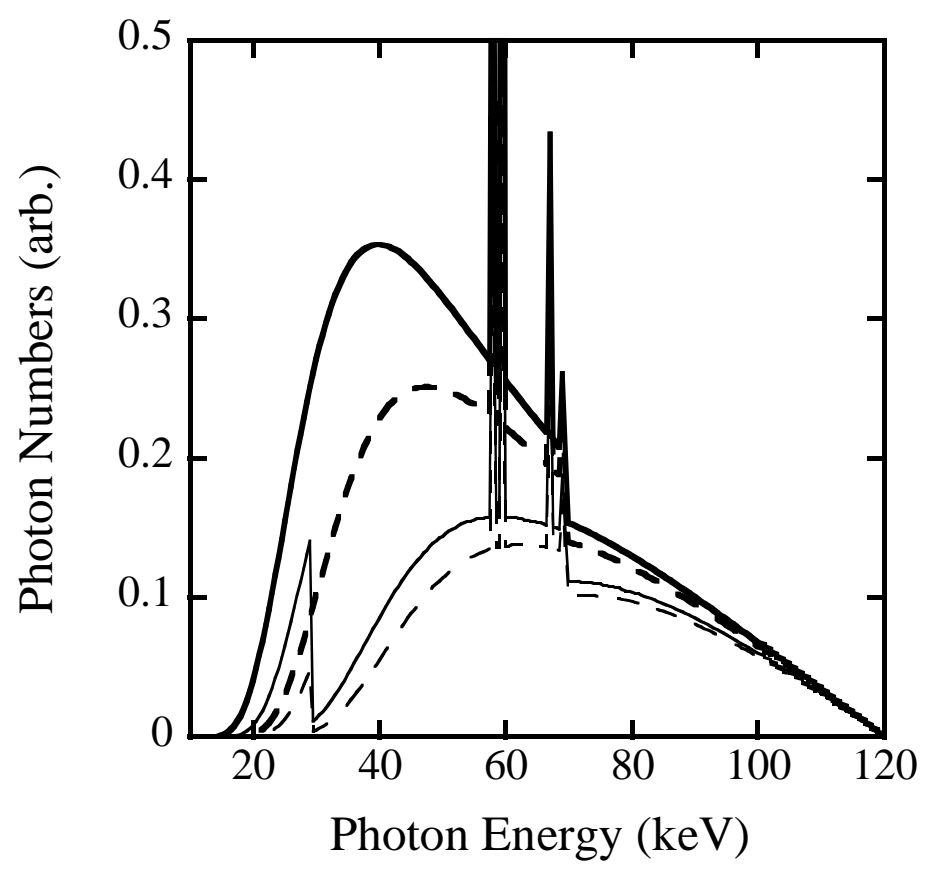

Figure 3 
Figure 4

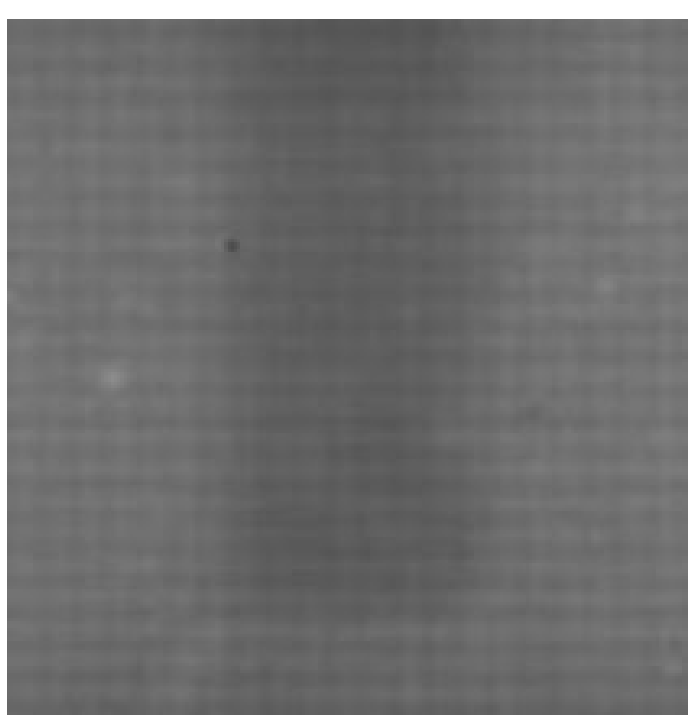



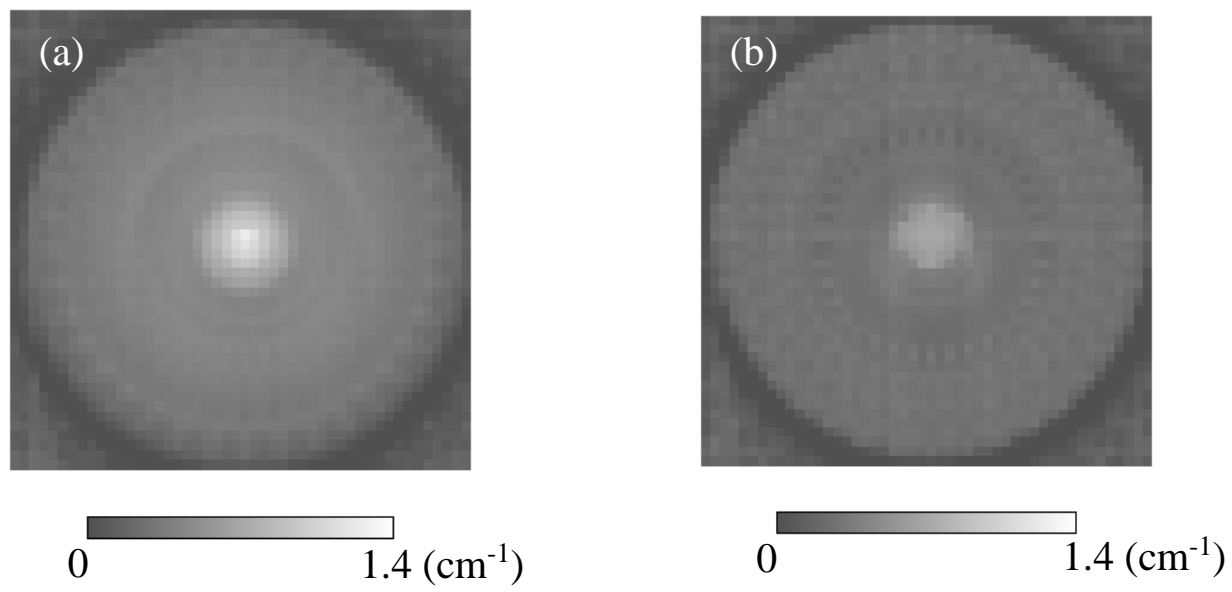

Figure 5 


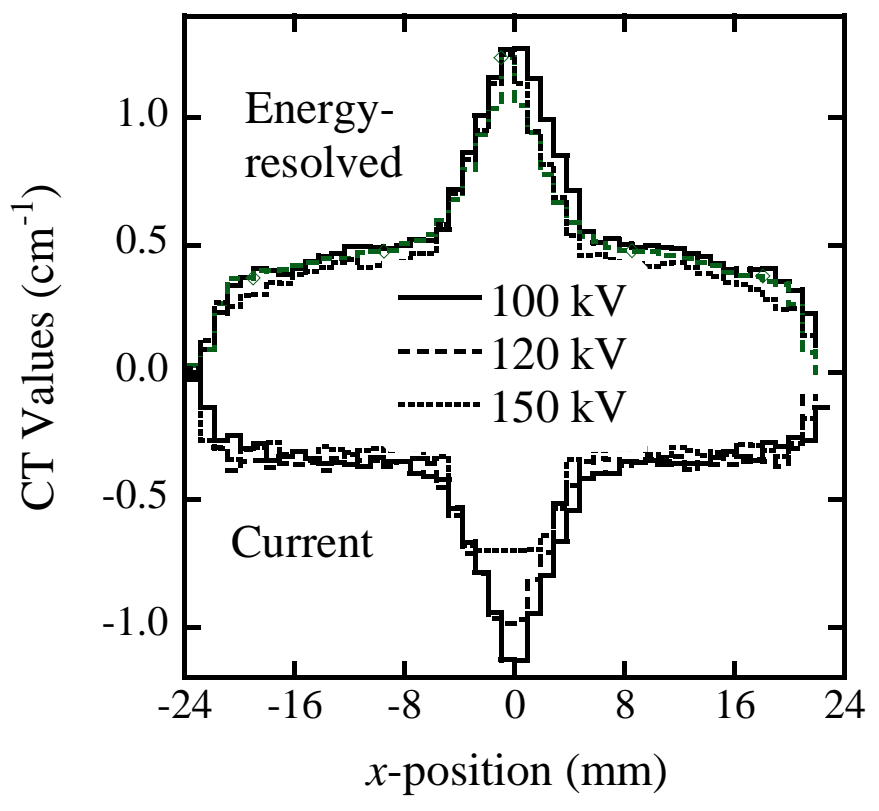

Figure 6 


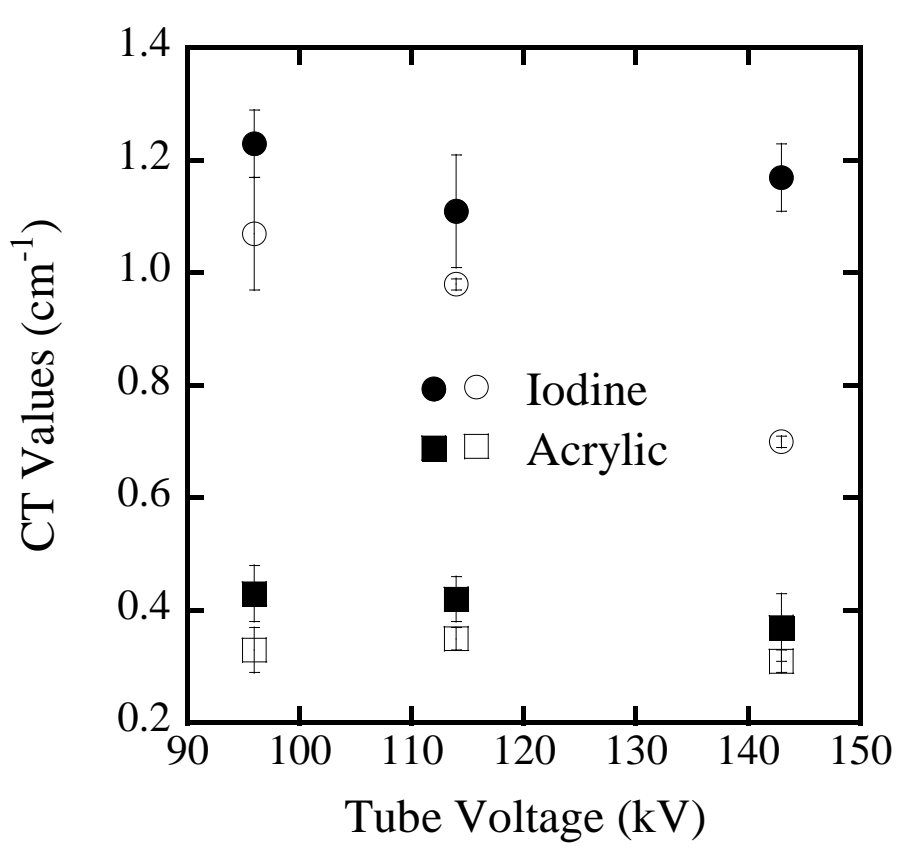

Figure 7 